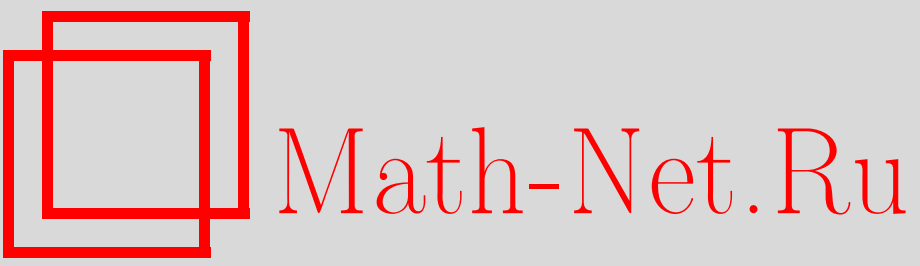

С. Н. Мартынов, Ангармонические состояния одномерной анизотропной модели Гейзенберга со свободными граничными условиями, ТМФ, 1998, том 117, номер 3, 489-497

DOI: https://doi.org/10.4213/tmf947

Использование Общероссийского математического портала Math-Net.Ru подразумевает, что вы прочитали и согласны с пользовательским соглашением

http: //www . mathnet.ru/rus/agreement

Параметры загрузки:

IP : 18.208 .226 .222

26 апреля 2023 г., 16:37:24 


\section{АНГАРМОНИЧЕСКИЕ СОСТОЯНИЯ ОДНОМЕРНОЙ АНИЗОТРОПНОЙ МОДЕЛИ ГЕЙЗЕНБЕРГА СО СВОБОДНЫМИ ГРАНИЧНЫМИ УСЛОВИЯМИ}

Для одномерной анизотропной модели Гейзенберга с конечным числом спинов $1 / 2$ и свободными граничными условиями показано существование состояний, описываемых негармоническими функциями. Определено число таких состояний.

\section{1. ВВЕДЕНИЕ}

Решения одномерной модели Гейзенберга имеют принципиальное значение в теории магнетизма. Это обусловлено большим числом задач, на которые возможно обобшение этой модели. Кроме того, ее точные решения являются критериями адекватности большого числа приближенных подходов и численных методов, применяемых в случаях, если нахождение точных решений невозможно. Собственные функции и собственные значения энергии модели Гейзенберга, как и любой другой модели, сушественно зависят не только от параметров гамильтониана, но и от граничных условий (ГУ), накладываемых на решения. Стандартные циклические ГУ $[1,2]$, позволяюшие использовать анзац Бете [3], ограничивают класс решений периодическими функциями координаты. Однако на практике, как правило, реализуется случай свободных ГУ, когда крайние спины магнитной цепочки взаимодействуют только с одним ближайшим магнитным соседом. Рассмотрение открытой анизотропной цепочки впервые было проведено Годеном [2]. Были получены обшие соотношения на импульсы и фазы для произвольного числа $M$ перевернутых спинов и сделано предположение о сушествовании вешественных решений для импульсов. Однако вопрос об анализе условий появления мнимых решений $k_{i}$ и их числа остался открытым. Для решения этого вопроса найдем полный набор собственных функций и собственных значений энергии для $M=1,2$ и для случая анизотропии обмена типа "легкая ось".

\section{2. ОДНОМАГНОННЫЕ РЕШЕНИЯ}

Анизотропная модель Гейзенберга с конечным числом $N$ спинов $S=1 / 2$ задается гамильтонианом

$$
H=J \sum_{n}\left(S_{n}^{z} S_{n+1}^{z}+\Delta\left(S_{n}^{x} S_{n+1}^{x}+S_{n}^{y} S_{n+1}^{y}\right)\right)
$$

\footnotetext{
* Институт физики им. Л.В. Киренского СО РАН, Красноярск, Россия
} 
В классе одномагнонных состояний $M=1$ с собственными функциями

$$
\psi(k)=\sum_{n=-(N-1) / 2}^{(N-1) / 2} a_{k}(n) \psi(n),
$$

где $n$ - координата перевернутого спина, отсчитываемая от середины магнитной цепочки, $k$ - импульс, параметризуюший решения, может быть найдено точное решение соответствуюшего уравнения Шредингера, которое для амплитуд $a_{k}(n)$ сводится к системе конечно-разностных уравнений

$$
2(\varepsilon+1) a(n)=\Delta(a(n-1)+a(n+1)), \quad-\frac{N-1}{2}<n<\frac{N-1}{2} .
$$

Координата $n$ принимает целые значения для нечетного $N$ и полуцелые- для четного $N$. Здесь и далее индекс $k$ у амплитуд $a(n)$ опушен для упрощения записи формул. Все выражения для амплитуд относятся к собственным состояниям (1) с фиксированным $k$. Энергия $\varepsilon=E / J$ отсчитывается от ферромагнитного "вакуума" в единицах $J$. В случае свободных ГУ для крайних амплитуд конечно-разностное уравнение имеет вид

$$
2\left(\varepsilon+\frac{1}{2}\right) a\left( \pm \frac{N-1}{2}\right)=\Delta a\left( \pm \frac{N-3}{2}\right) .
$$

Эта исходная неоднородная система конечно-разностных уравнений (3) и (4) эквивалентна однородной системе, если дополнить набор из $N$ физических амплитуд $a(n)$, $n \in(-(N-1) / 2, \ldots,(N-1) / 2)$, двумя нефизическими амплитудами, связанными с физическим набором, двумя дополнительными условиями

$$
a\left( \pm \frac{N-1}{2}\right)=\Delta a\left( \pm \frac{N+1}{2}\right) .
$$

Почленное добавление условий (5) к граничным уравнениям (4) сводит задачу к решению однородной системы конечно-разностных уравнений, на которую наложены ГУ (5). Эти условия для $\Delta \neq 1$ уже не являются условиями зеркального типа, обеспечивавшими периодичность решений для изотропной модели Гейзенберга [4]. Нециклические ГУ любой конечной системы требуют обрашения в нуль физической амплитуды волновой функции за границами области определения. Это позволяет искать решения (с точностью до несущественного фазового множителя) в виде реальных функций координат с симметрией, отражаюшей симметрию рассматриваемой задачи. Гармонические симметричные (s) и антисимметричные (a) относительно середины цепочки спинов $n=0$ решения однородной системы (3), (4), (5) с учетом нормировки имеют соответственно вид

$$
\begin{aligned}
& a_{k}^{\mathrm{S}}(n)=\sqrt{\frac{2}{N+\frac{\sin (k N)}{\sin k}}} \cos (k n), \\
& a_{k}^{\mathrm{a}}(n)=\sqrt{\frac{2}{N-\frac{\sin (k N)}{\sin k}}} \sin (k n) .
\end{aligned}
$$


Волновые векторы решений (6) определяются ГУ (5)

$$
\begin{aligned}
\operatorname{ctg} \frac{k N}{2} & =-\frac{1+\Delta}{1-\Delta} \operatorname{tg} \frac{k}{2}, \\
\operatorname{tg} \frac{k N}{2} & =\frac{1+\Delta}{1-\Delta} \operatorname{tg} \frac{k}{2}
\end{aligned}
$$

соответственно для симметричных и антисимметричных решений. Решения $k_{j}$ уравнений для состояний разной симметрии дают функции (2), ортогональные по определению. Решения одинаковой симметрии удовлетворяют условиям ортогональности

$$
\frac{\sin \frac{N\left(k+k^{\prime}\right)}{2}}{\sin \frac{k+k^{\prime}}{2}} \pm \frac{\sin \frac{N\left(k-k^{\prime}\right)}{2}}{\sin \frac{k-k^{\prime}}{2}}=0
$$

для симметричных и антисимметричных пар $k$ и $k^{\prime}$, соответственно. В области определения физически различных решений $0 \leqslant k<\pi$ число решений уравнения (7) для четного $N$ есть

$$
N_{1 m}^{\mathrm{s}}=\frac{N}{2}-1 \text {. }
$$

Число антисимметричных гармонических решений (8) зависит от числа спинов $N$ и величины $\Delta$ :

Для нечетного $N$ получаем соответственно

$$
N_{1 m}^{\mathrm{a}}= \begin{cases}\frac{N}{2}, & N<\frac{1+\Delta}{1-\Delta}, \\ \frac{N}{2}-1, & N>\frac{1+\Delta}{1-\Delta} .\end{cases}
$$

$$
\begin{aligned}
& N_{1 m}^{\mathrm{s}}=\frac{N-1}{2}, \\
& N_{1 m}^{\mathrm{a}}= \begin{cases}\frac{N-1}{2}, & N<\frac{1+\Delta}{1-\Delta}, \\
\frac{N-1}{2}-1, & N>\frac{1+\Delta}{1-\Delta} .\end{cases}
\end{aligned}
$$

Случай

$$
N=\frac{1+\Delta}{1-\Delta}
$$

является особым. Здесь мы имеем линейное антисимметричное решение

$$
a_{l}^{\mathrm{a}}(n)=\frac{2 \sqrt{3}}{\sqrt{N\left(N^{2}-1\right)}} n
$$

с энергией дна одномагнонной ветви $\varepsilon_{l}=\Delta-1$. Таким образом, гармонические одномагнонные решения (6) дают число $k$-состояний, меньшее чем исходное число $N$ координатных функций. Полный набор ортонормированных одномагнонных функций можно получить расширением класса экспоненциальных функций (6) ангармоническими гиперболическими функциями соответствуюшей симметрии, что отвечает решениям с мнимыми импульсами $k=i q$ :

$$
\begin{aligned}
& a_{q}^{\mathrm{s}}(n)=\sqrt{\frac{2}{N+\frac{\operatorname{sh}(q N)}{\operatorname{sh} q}}} \operatorname{ch}(q n), \\
& a_{q}^{\mathrm{a}}(n)=\sqrt{\frac{2}{\frac{\operatorname{sh}(q N)}{\operatorname{sh} q}-N}} \operatorname{sh}(q n) .
\end{aligned}
$$




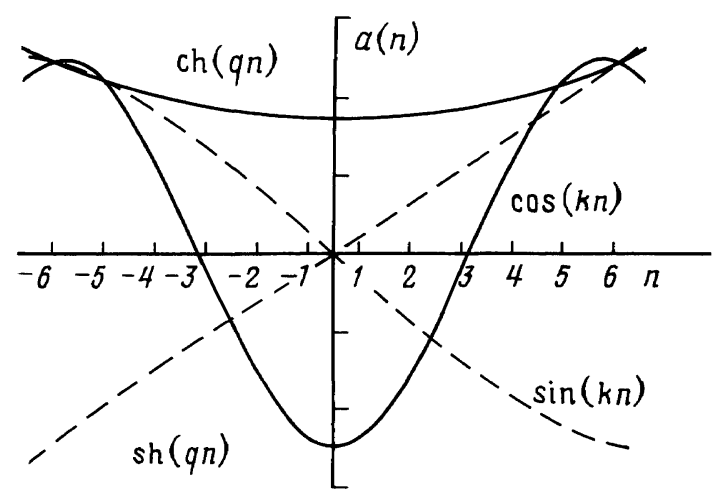

Рис. 1

Соответствующие уравнения для $q$ опять получаются подстановкой соотношений (10) в ГУ (5) и имеют вид

$$
\begin{aligned}
\operatorname{cth} \frac{q N}{2} & =\frac{1+\Delta}{1-\Delta} \operatorname{th} \frac{q}{2}, \\
\operatorname{th} \frac{q N}{2} & =\frac{1+\Delta}{1-\Delta} \operatorname{th} \frac{q}{2}
\end{aligned}
$$

соответственно для симметричного и антисимметричного случаев. Видно, что симметричное ангармоническое решение существует при любых $N$ и $\Delta<1$, в то время как равенство для антисимметричных решений (12) имеет место только при выполнении порогового условия

$$
N>\frac{1+\Delta}{1-\Delta}
$$

Таким образом, сумма гармонических и ангармонических одномагнонных решений всегда обеспечивает полноту системы собственных функций оператора (1).

Вид различных одномагнонных решений с минимальными значениями $k$ и $q$ для $N=$ 10 и $\Delta=0.9$ показан на рис. 1 . Для пояснения порогового условия (13) функции взяты ненормированными, но проходяшими через одни и те же граничные точки (5). Видно, что при заданных $N$ и $\Delta$ антисимметричное негармоническое решение не может проходить через эти точки и центр инверсии, т.е. такое решение отсутствует. Для реализации такого решения необходимо либо увеличить $N$, либо уменьшить $\Delta$. При этом исчезнет первое антисимметричное гармоническое решение $a \sim \sin (k n)$.

\section{3. ДВУХМАГНОННЫЕ РЕШЕНИЯ}

Для $M=2$ требования симметрии, накладываемые на амплитуды собственных функций

$$
\psi\left(k_{1}, k_{2}\right)=\sum_{n_{1}<n_{2}} a\left(n_{1}, n_{2}\right) \psi\left(n_{1}, n_{2}\right),
$$

сводятся к условиям соответствующей четности амплитуд относительно инверсии координат перевернутых спинов

$$
a\left(n_{1}, n_{2}\right)= \pm a\left(-n_{2},-n_{1}\right) .
$$


Здесь и далее индексы $k_{1}, k_{2}$ у амплитуд опущены для упрошения выражений. Все амплитуды $a\left(n_{1}, n_{2}\right)$ относятся к фиксированной паре $k_{1}, k_{2}$, параметризуюшей каждое двухмагнонное решение. Знак плюс имеет место для функций с симметричным относительно центра распределением амплитуд, а минус - для антисимметричного распределения. Неоднородная система конечно-разностных уравнений на амплитуды сводится к однородной системе

$$
\begin{aligned}
2(\varepsilon+2) a\left(n_{1}, n_{2}\right)=\Delta & \left(a\left(n_{1}+1, n_{2}\right)+a\left(n_{1}-1, n_{2}\right)+\right. \\
& \left.+a\left(n_{1}, n_{2}+1\right)+a\left(n_{1}, n_{2}-1\right)\right), \quad n_{1}<n_{2},
\end{aligned}
$$

путем дополнения физических амплитуд $a\left(n_{1}<n_{2}\right)$,

$$
n_{1}, n_{2} \in\left(-\frac{N-1}{2}, \ldots, \frac{N-1}{2}\right)
$$

$3 N-2$ нефизическими амплитудами $a(n, n), a\left(-(N+1) / 2, n_{2}\right), a\left(n_{1},(N+1) / 2\right)$, на которые накладываются граничные условия

$$
\begin{gathered}
a\left(-\frac{N-1}{2}, n_{2}\right)=\Delta a\left(-\frac{N+1}{2}, n_{2}\right), \\
a\left(n_{1}, \frac{N-1}{2}\right)=\Delta a\left(n_{1}, \frac{N+1}{2}\right), \\
2 a_{k_{1} k_{2}}\left(n_{1}, n_{1}+1\right)=\Delta\left(a_{k_{1} k_{2}}\left(n_{1}, n_{1}\right)+a_{k_{1} k_{2}}\left(n_{1}+1, n_{1}+1\right)\right) .
\end{gathered}
$$

Процедура полностью аналогична изотропному случаю [4] и обеспечивает равенство полного числа амплитуд и уравнений, необходимое для выполнения условия однозначности решения. С учетом вешественности амплитуд получаем для гармонических решений соотношение

$$
\begin{aligned}
a^{\mathrm{s}, \mathrm{a}}\left(n_{1}, n_{2}\right)= & C\left(k_{1}, k_{2}\right)\left(\cos \left(k_{1}\left(n_{1}+\delta_{1}\right)\right) \cos \left(k_{2}\left(n_{2}-\delta_{2}\right)\right) \pm\right. \\
& \left. \pm \cos \left(k_{2}\left(n_{1}+\delta_{2}\right)\right) \cos \left(k_{1}\left(n_{2}-\delta_{1}\right)\right)\right) .
\end{aligned}
$$

Фазовые сдвиги $\delta_{1}=\delta\left(k_{1}\right), \delta_{2}=\delta\left(k_{2}\right)$ задают положения максимумов волновой функции, и для зеркальных ГУ (изотропная модель Гейзенберга) они равны $N / 2$ [4]. Для анизотропного случая они определяются из ГУ (16) с помощью уравнений

$$
\delta_{i}=\delta_{i}^{\prime}+\frac{N}{2}, \quad \operatorname{ctg}\left(k_{i} \delta_{i}^{\prime}\right)=\frac{1+\Delta}{1-\Delta} \operatorname{tg} \frac{k_{i}}{2}, \quad i=1,2 .
$$

Связь между импульсами $k_{1}$ и $k_{2}$ каждого решения задается соотношением (17), которое устанавливает взаимно однозначное соответствие между $k_{1}$ и $k_{2}$, т.е. двухмагнонные решения анизотропной одномерной модели Гейзенберга являются однопараметрическими. Для симметричных и антисимметричных решений получаем соответственно две системы уравнений:

$$
\begin{gathered}
\frac{\sin 2 k_{1} \delta_{1}}{\sin k_{1}}\left(1-\Delta \cos k_{1}\right) \pm \frac{\sin 2 k_{2} \delta_{2}}{\sin k_{2}}\left(1-\Delta \cos k_{2}\right)=0, \\
\frac{\sin k_{1}\left(2 \delta_{1}-1\right)}{\sin k_{1}} \pm \frac{\sin k_{2}\left(2 \delta_{2}-1\right)}{\sin k_{2}}=0,
\end{gathered}
$$


где $\delta_{1,2}$ заданы соотношениями (19).

При рассмотрении вешественных решений $k_{i}$ удобнее исключить фазы (19) и использовать уравнения для полного $K=k_{1}+k_{2}$ и относительного $q=\left(k_{1}-k_{2}\right) / 2$ импульсов. Имеем

$$
\begin{aligned}
& \operatorname{tg}\left(\frac{K N}{2}\right) \sin \left(\frac{K}{2}\right)\left(2 \cos \frac{K}{2}-\Delta\left(3-\Delta^{2}\right) \cos q\right)+\Delta^{2}-1+ \\
& +2 \cos ^{2} \frac{K}{2}+2 \Delta^{2} \cos ^{2} q-\Delta\left(3+\Delta^{2}\right) \cos \left(\frac{K}{2}\right) \cos q=0 \\
& \operatorname{tg}(q N) \sin (q)\left(2 \cos q-\Delta\left(3-\Delta^{2}\right) \cos \frac{K}{2}\right)+\Delta^{2}-1+ \\
& +2 \cos ^{2} q+2 \Delta^{2} \cos ^{2} \frac{K}{2}-\Delta\left(3+\Delta^{2}\right) \cos \left(\frac{K}{2}\right) \cos q=0
\end{aligned}
$$

для симметричных решений и

$$
\begin{aligned}
& \operatorname{ctg}\left(\frac{K N}{2}\right) \sin \left(\frac{K}{2}\right)\left(\Delta\left(3-\Delta^{2}\right) \cos q-2 \cos \frac{K}{2}\right)+\Delta^{2}-1+ \\
& +2 \cos ^{2} \frac{K}{2}+2 \Delta^{2} \cos ^{2} q-\Delta\left(3+\Delta^{2}\right) \cos \left(\frac{K}{2}\right) \cos q=0 \\
& \operatorname{ctg}(q N) \sin (q)\left(\Delta\left(3-\Delta^{2}\right) \cos \frac{K}{2}-2 \cos q\right)+\Delta^{2}-1+ \\
& \quad+2 \cos ^{2} q+2 \Delta^{2} \cos ^{2} \frac{K}{2}-\Delta\left(3+\Delta^{2}\right) \cos \left(\frac{K}{2}\right) \cos q=0
\end{aligned}
$$

для антисимметричных решений.

Уравнения (20) инвариантны относительно инверсии знаков $k_{i}$ и их перестановок (знаков и перестановок $K$ и $q$ в (20), (21)). Для определения всех физически различных решений $(20)$ и (21) достаточно ограничиться интервалом $0 \leqslant K, q<\pi$. Кроме вешественных решений $K$ и $q$, уравнения имеют решения, когда один из этих импульсов мнимый. Симметрия уравнений при перестановках $K$ и $q$ позволяет получить полный набор таких физически различных состояний, фиксируя $K$ вешественным, а $q$ мнимым. Эти решения соответствуют связанным комплексам Бете $k_{1}=k_{2}^{*}$, и для них уравнения (20), (21) для $K$ и $q^{\prime}=-i q$ по-прежнему остаются вешественными. Амплитуды комплексов Бете в координатах $R=\left(n_{1}+n_{2}\right) / 2$ и $r=n_{1}-n_{2}$ имеют вид

$$
\begin{aligned}
a_{B}^{\mathrm{s}}(R, r)= & C\left(K, q^{\prime}\right)\left(\cos (K R) \operatorname{ch}\left(q^{\prime}\left(r+N+2 \operatorname{Re}\left(\delta^{\prime}\right)\right)+K \operatorname{Im}\left(\delta^{\prime}\right)\right)+\right. \\
& \left.+\operatorname{ch}\left(2 q^{\prime} N\right) \cos \left(\frac{K}{2}\left(r+N+2 \operatorname{Re}\left(\delta^{\prime}\right)\right)+2 q^{\prime} \operatorname{Im}\left(\delta^{\prime}\right)\right)\right) \\
a_{B}^{\mathrm{a}}(R, r)= & C\left(K, q^{\prime}\right)\left(\sin (K R) \operatorname{sh}\left(q^{\prime}\left(r+N+2 \operatorname{Re}\left(\delta^{\prime}\right)\right)+K \operatorname{Im}\left(\delta^{\prime}\right)\right)+\right. \\
& \left.+\operatorname{sh}\left(2 q^{\prime} N\right) \sin \left(\frac{K}{2}\left(r+N+2 \operatorname{Re}\left(\delta^{\prime}\right)\right)+2 q^{\prime} \operatorname{Im}\left(\delta^{\prime}\right)\right)\right)
\end{aligned}
$$


для симметричных и антисимметричных комплексов, соответственно, где $\delta^{\prime}=$ $\delta_{1}^{\prime}=\left(\delta_{2}^{\prime}\right)^{*}$.

Вешественные и комплексно-сопряженные решения $k_{i}$ для анизотропной открытой цепочки $\Delta<1$ не исчерпывают все возможные решения уравнений (20) и не дают полного набора собственных функций (1). Уравнения (20) имеют решения и в случае, если один из импульсов $k_{i}$ или оба сразу являются чисто мнимыми. В первом случае реализуются смешанные состояния (m-состояния), когда амплитуды волновых функций выражаются через суперпозищию гармонических (тригонометрических) и гиперболических функций:

$$
\begin{aligned}
a_{\mathrm{m}}^{\mathrm{s}, \mathrm{a}}\left(n_{1}, n_{2}\right)= & C\left(k_{1}, k_{2}\right)\left(\cos \left(k_{1}\left(n_{1}+\delta_{1}\right)\right) \operatorname{ch}\left(\left|k_{2}\right|\left(n_{2}-\delta_{1}\right)\right) \pm\right. \\
& \left. \pm \operatorname{ch}\left(\left|k_{2}\right|\left(n_{1}+\delta_{2}\right)\right) \cos \left(k_{1}\left(n_{2}-\delta_{1}\right)\right)\right) .
\end{aligned}
$$

Во втором случае получаем чисто ангармонические состояния (а-состояния), у которых амплитуды выражаются через гиперболические функции:

$$
\begin{aligned}
a_{\mathrm{a}}^{\mathrm{s}}\left(n_{1}, n_{2}\right)= & C\left(k_{1}, k_{2}\right)\left(\operatorname{ch}\left(\left|k_{1}\right|\left(n_{1}+\delta_{1}\right)\right) \operatorname{ch}\left(\left|k_{2}\right|\left(n_{2}-\delta_{2}\right)\right)+\right. \\
& \left.+\operatorname{ch}\left(\left|k_{2}\right|\left(n_{1}+\delta_{2}\right)\right) \operatorname{ch}\left(\left|k_{1}\right|\left(n_{2}-\delta_{1}\right)\right)\right), \\
a_{\mathrm{a}}^{\mathrm{a}}\left(n_{1}, n_{2}\right)= & C\left(k_{1}, k_{2}\right)\left(\operatorname{ch}\left(\left|k_{1}\right|\left(n_{1}+\delta_{1}\right)\right) \operatorname{sh}\left(\left|k_{2}\right|\left(n_{2}-\delta_{2}\right)\right)+\right. \\
& \left.+\operatorname{sh}\left(\left|k_{2}\right|\left(n_{1}+\delta_{2}\right)\right) \operatorname{ch}\left(\left|k_{1}\right|\left(n_{2}-\delta_{1}\right)\right)\right) .
\end{aligned}
$$

Граничные условия (16) для амплитуд $a_{\mathrm{a}}^{\mathrm{a}}\left(n_{1}, n_{2}\right)$ дают вместо $(19)$ следующее выражение для фазы $\delta_{2}$ :

$$
\delta_{2}=\delta_{2}^{\prime}+\frac{N}{2}, \quad \operatorname{th}\left(\left|k_{2}\right| \delta_{2}^{\prime}\right)=-\frac{1+\Delta}{1-\Delta} \operatorname{th} \frac{k_{2}}{2} .
$$

\section{4. ЭНЕРГИИ ОДНО- И ДВУХМАГНОННЫХ СОСТОЯНИЙ}

Спектр собственных значений энергии рассмотренных решений в зависимости от суммарного вешественного импульса $K=\operatorname{Re}\left(\sum k_{i}\right)$ показан для $N=10$ и $\Delta=0.9$ на рис. 2 . Одномагнонным состояниям соответствуют кружки (темные для симметричных и светлые для антисимметричных состояний). Квадраты отвечают двухмагнонным состояниям (темные симметричным, светлые антисимметричным). Одномагнонные энергии содержат $N-1$ волновых и одно ангармоническое значения

$$
\begin{aligned}
& \varepsilon_{\mathrm{w}}^{(1)}=\Delta \cos k-1, \\
& \varepsilon_{\mathrm{a}}^{(1)}=\Delta \operatorname{ch} q-1 .
\end{aligned}
$$

Последнее относится к симметричному решению. Пороговое условие (13) для данного примера не выполнено и антисимметричное а-состояние отсутствует. Поскольку $\operatorname{ch} q \geqslant 1$, энергии ангармонических решений для ферромагнитного обмена $J<0$ лежат ниже ветви волновых энергий. Причем энергия симметричного ангармонического состояния минимальная (первое возбужденное состояние). Этот вывод прямо следует из 


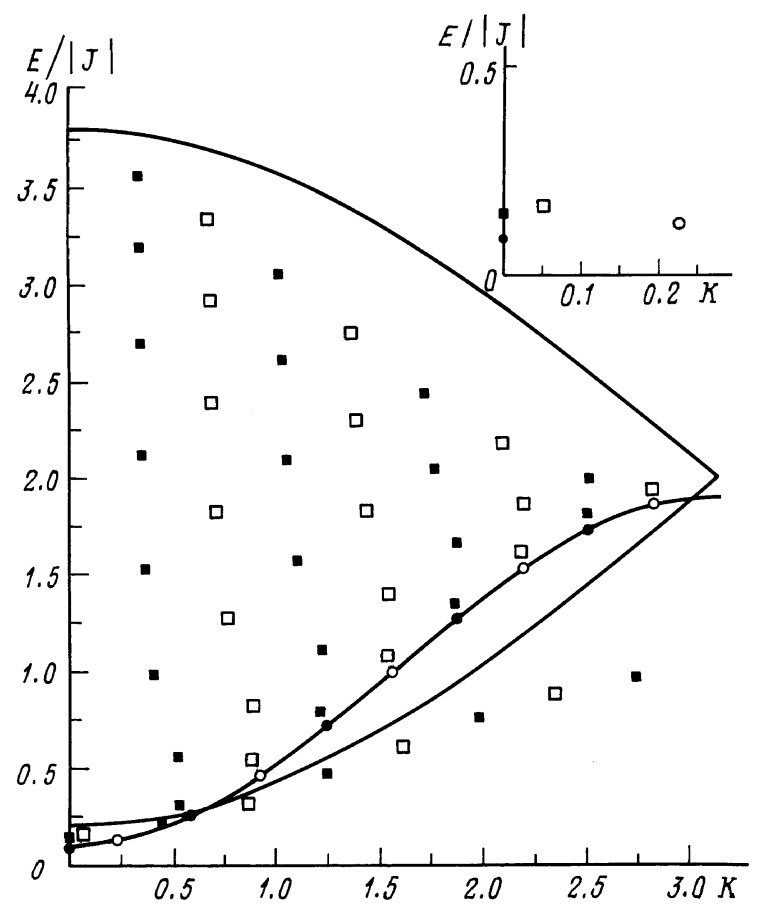

Рис. 2

вида волновых функций (см. рис. 1): энергия состояния тем ниже, чем более однородным является распределение намагниченности (амплитуды волновой функции) по цепочке. Распределение уровней в данной задаче полностью аналогично распределению уровней частищы в потенциальной яме в зависимости от числа узлов волновой функции.

Волновые двухмагнонные решения дают значения энергии

$$
\varepsilon_{w}^{(2)}=\Delta\left(\cos k_{1}+\cos k_{2}\right)-2,
$$

число которых по сравнению с изотропным случаем [4] уменьшается на $N$. Это уменьшение происходит за счет появления $N \mathrm{~m}$ - и а-решений. Для данного примера сушествует только одно симметричное а-решение, имеющее наинизшую энергию среди двухмагнонных значений (на вставке рис. 2 оно находится на оси $K=0$ ). Антисимметричное а-решение появляется при увеличении анизотропии (для $N=10)$ до $\Delta \approx 0.8$ за счет одного антисимметричного т-решения (на вставке рис. 2 оно ближайшее к оси $K=0$ ). Энергии смешанных и ангармонических решений имеют соответственно вид

$$
\begin{aligned}
& \varepsilon_{\mathrm{m}}^{(2)}=\Delta\left(\cos k_{1}+\operatorname{ch}\left|k_{2}\right|\right)-2, \\
& \varepsilon_{\mathrm{a}}^{(2)}=\Delta\left(\operatorname{ch}\left|k_{1}\right|+\operatorname{ch}\left|k_{2}\right|\right)-2 .
\end{aligned}
$$

Комплексы Бете образуют нижнюю ветвь двухмагнонных энергий

$$
\varepsilon_{\mathrm{B}}^{(2)}=2 \Delta \cos K \operatorname{ch}|q|-2
$$




\section{5. ОБСУЖДЕНИЕ}

Обшие соотношения на импульсы и фазы экспоненциальных функций Бете [2] позволяют для $M=1$ после соответствуюших преобразований получить решения (6)-(8). Расширение области допустимых значений импульсов в область мнимых $k$ дает ангармонические состояния, дополняюшие набор до $N$. Число последних (1 или 2) зависит от соотношения между числом спинов и анизотропией, а энергия является наинизшей. Линейное решение, возникаюшее при $N=(1+\Delta) /(1-\Delta)$, не может быть получено из конечного числа экспонент. Это говорит о том, что экспоненты не всегда дают полный набор собственных функций.

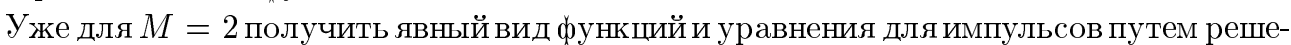
ния общих соотношений [2] весьма сложно. Использование симметризованных вещественных волновых функций позволяет математически просто и физически наглядно решить задачу анализа возможных типов решений и их числа. Анизотропия обмена $\Delta<1$ (“легкая ось”) в сочетании со свободными ГУ приводит к уменьшению числа решений с вешественными $k_{i}$ и появлению за их счет $N$ решений с мнимыми $k_{i}$. Анизотропия $\Delta>1$ (“легкая плоскость") не приводит к появлению таких решений. В этом случае основное состояние вырождено по направлению в базисной плоскости и вид волновых функций возбужденных состояний требует отдельного рассмотрения. Число связанных комплексов Бете с появлением анизотропии $\Delta<1$ не меняется, хотя по-прежнему имеет нетривиальную зависимость от числа спинов $[5,6]$. Полное число состояний равно исходному числу состояний $C_{N}^{2}=N(N-1) / 2$, все состояния являются однопараметрическими, состояний с совпадающими импульсами нет. Для ферромагнитного обмена энергии а-состояний лежат ниже дна зоны волновых двухмагнонных энергий, а значения энергий $\mathrm{m}$-состояний образуют нижнюю двухмагнонную ветвь, проходящую над одномагнонной ветвью (см. рис. 2). Значения их вешественных импульсов $k_{j}$ близки к одномагнонным, но не равны им, как в изотропном случае. Это означает, что резонансные переходы $\Delta M=1$ возможны только с $\Delta K \neq 0$, и, поскольку в данном случае $\Delta K \ll \pi / N$, именно эта ветвь дает макроскопическую вероятность для переходов в однородном резонансном поле $W_{n} \sim N$.

\section{Список литературы}

[1] Ю.А. Изюмов, Ю. И. Скрябин. Статистическая механика магнитоупорядоченных систем. М.: Наука, 1987.

[2] М. Годен. Волновая функция Бете. М.: Мир, 1987.

[3] H. A. Bethe. Z. Phys. 1931. V. 71. P. 205.

[4] С. Н. Мартынов. ТМФ. 1997. Т. 113. С. 338.

[5] K. Isler, M. B. Paranjape. Phys. Lett. B. 1993. V. 319. P. 209.

[6] S. N. Martynov. Phys. Lett. A. 1996. V. 219. P. 329.

Поступила в редакцию 5.V.1998 г., после доработки 6.VII. 1998 г. 